\title{
Efeitos no aumento da incidência e a gravidade de lesões por pressão e atuação do enfermeiro: uma revisão integrativa
}

\author{
Effects on increasing the incidence and severity of pressure injuries and nurses' performance: an \\ integrative review
}

Efectos sobre el aumento de la incidencia y la gravedad de las lesiones por presión y el papel de las enfermeras: una revisión integradora

\section{Resumo}

Objetivo: realizar uma revisão integrativa sobre efeitos no aumento da incidência e a gravidade de úlceras por pressão e atuação do enfermeiro, transmitir conhecimento e despertar nos profissionais de enfermagem interesse em buscar qualificações a respeito de cuidados com lesão por pressão. Definir a necessidade de acompanhamento por um profissional de enfermagem, em pacientes com lesão por pressão. Metodologia: Trata-se de uma revisão integrativa da literatura, formulação da questão problema, fica definido a questão: "Efeitos no aumento da incidência e a gravidade de úlceras por pressão e atuação do enfermeiro uma revisão integrativa". Resultados: A vulnerabilidade e riscos à saúde dos pacientes, afetam nos indicadores de qualidade da assistência de enfermagem prestadas aos clientes. Conclusão: A importância da equipe multidisciplinar em destaque a Enfermagem ter conhecimento em relação a LPPs, e cuidados é o primeiro passo para realização de medidas de prevenção e tratamento, evitando agravos a saúde do paciente.

Palavras-chave: Lesão por pressão; Assistência de enfermagem; Prevenção; Escara de decúbito.

\begin{abstract}
Objective: to carry out an integrative review on the effects of increasing the incidence and severity of pressure ulcers and the role of nurses, transmitting knowledge and awakening in nursing professionals an interest in seeking qualifications regarding pressure ulcer care. Define the need for monitoring by a nursing professional in patients with pressure injuries. Methodology: This is an integrative literature review, formulation of the problem question, the question is defined: "Effects on the increase in the incidence and severity of pressure ulcers and the role of nurses in an integrative review". Results: The vulnerability and health risks of patients affect the quality indicators of nursing care provided to clients. Conclusion: The importance of the multidisciplinary team in Nursing highlighting knowledge about LPPs, and care is the first step in carrying out prevention and treatment measures, avoiding harm to the patient's health.
\end{abstract}

Keywords: Pressure injury; Nursing care; Prevention; Decubitus sores.

\section{Resumen}

Objetivo: realizar una revisión integradora sobre los efectos del aumento de la incidencia y gravedad de las úlceras por presión y el papel del enfermero, transmitiendo conocimientos y despertando en los profesionales de enfermería el interés por la búsqueda de titulaciones en el cuidado de las úlceras por presión. Definir la necesidad de seguimiento por parte de un profesional de enfermería en pacientes con lesiones por presión. Metodología: Se trata de una revisión integradora de la literatura, formulación de la pregunta problema, se define la pregunta: "Efectos sobre el aumento de la incidencia y gravedad de las úlceras por presión y el papel del enfermero en Resultados: La vulnerabilidad y los riesgos para la salud de los pacientes afectan los indicadores de calidad de la atención de enfermería brindada a los clientes. Conclusión: La importancia del equipo multidisciplinario en Enfermería resaltando el conocimiento sobre las PLP y el cuidado es el primer paso para llevar a cabo las medidas de prevención y tratamiento, evitando dañar la salud del paciente.

Palabras clave: Lesión por presión; Atención de enfermeía; Prevención; Úlceras por decúbito. 


\section{Introdução}

A incidência de úlceras por pressão é um problema mundial de saúde e se encontra em os seis eventos adversos em saúde que comumente acontece. Estudos estão sendo realizados para a compreensão de como acontece o desenvolvimento da lesão por pressão (LPP), fatores contribuintes são, pressão, cisalhamento, fricção e humidade (Gamston, 2019).

O ministério da saúde instituiu, no Brasil, em 2013, a Portaria n 529/2013, o Programa Nacional de Segurança do Paciente (PNSP), promovendo a proteção do paciente e segurança em todos os serviços de saúde, a gestão dos riscos e comprometimento dos profissionais (Brasil, 2013).

A escala de Braden utilizada na prática clínica, está ligada positivamente ao gerenciamento de risco e a prevenção durante o processo de cuidado realizado pela equipe de Enfermagem. A incidência das LPPs, em consequência altos custos, aumento de tempo de internação, prognósticos negativos e índices negativos da qualidade de assistência (JESUS et al., 2020).

O presente artigo se justifica pelo impacto considerável na qualidade de vida dos indivíduos acometidos, por Lesão por pressão (LPP), causando mudanças em sua rotina diária, provocando isolamento social, ocasionando dor, restrições físicas e isolamento social. O profissional de enfermagem tem atuação de grande importância relacionado a lesão por pressão, dispõem de uma considerável experiência clínica, emissores de conhecimento, na identificação, prevenção, no tratamento e implementação de técnicas para diminuição das lesões teciduais da pele (Woodhouse et al., 2019).

Se justifica pela relevância da prevenção de riscos e agravos à saúde, pertinente em evidenciar a necessidade de autonomia da atuação dos profissionais de enfermagem. Em especifico o estudo tem o intuito de gerar impacto na sociedade cientifica e promover conhecimento, contribuir favoravelmente para atualização e conscientização dos profissionais de enfermagem.

Dentre outros problemas, como o efeito da falha das políticas públicas de gestão em saúde à respeito dos pacientes em vulnerabilidade, elevados indicadores de infecção hospitalar e o ocasionalmente de óbitos. As lesões por pressão impactaram de forma relevante a saúde da população brasileira. O papel do profissional de enfermagem é relevante para os cuidados em unidade de terapia intensiva em relação a crianças, adultos e idosos com lesão por pressão.

Os profissionais de saúde atuantes em hospitais, precisam realizar especialização a respeito de cuidados com lesão por pressão. Os acadêmicos de enfermagem possuem pouco conhecimento no que diz a respeito à cuidados com lesão por pressão. A educação continuada é utilizada de maneira consistente em grandes centros hospitalares no Brasil. Quais os principais impactos da saúde na incidência e a gravidade de úlceras por pressão, e quais as contribuições do enfermeiro em sua atuação nos serviços de saúde e na comunidade acadêmica?

Tem como objetivo realizar uma revisão integrativa sobre efeitos no aumento da incidência e a gravidade de úlceras por pressão e atuação do enfermeiro, transmitir conhecimento e despertar nos profissionais de enfermagem interesse em buscar qualificações a respeito de cuidados com lesão por pressão. Definir a necessidade de acompanhamento por um profissional de enfermagem, em pacientes com lesão por pressão. Classificar os grupos de risco e vulnerabilidade. Os riscos para ocorrência e a gravidade de úlceras por pressão. Interpretar o grau de instruções dos profissionais de enfermagem em prevenção e promoção de saúde a respeito de lesão por pressão. Criar mecanismos de divulgação e comunicação para enfermeiros e comunidade como importante auxílio em medidas profiláticas para controle da lesão por pressão.

\section{Metodologia}

Trata-se de uma revisão integrativa da literatura, estudo com critérios de inclusão ou exclusão, o plano de pesquisa, análise da qualidade dos trabalhos científicos, incluindo estudos que respondia à pergunta tema da pesquisa, critérios incluindo língua e ano de apresentação (Estrela, 2018). 
Para compreensão completa da problemática estudada, fez-se necessário definir o conhecimento moderno sobre o conteúdo característico, determinado em fases, (1) Formulação da questão problema, (2) investigação e pesquisa dos trabalhos científicos, (3) busca de fatos, (4) critérios de inclusão, (5) compreensão e exposição dos resultados, (6) expor a revisão integrativa (SOUZA et al., 2010).

Formulação da questão problema, fez-se necessário escolha de uma determinada área de pesquisa com ênfase na atuação do enfermeiro, fatores que causam impacto social e econômico, escolha das determinantes atualizadas para realização da pesquisa nas bases de dados, fica definido a questão: "Efeitos no aumento da incidência e a gravidade de úlceras por pressão e atuação do enfermeiro uma revisão integrativa".

Para busca dos artigos na literatura, tais como: National Institutes of Health (PUBMED), Web of Science, Biblioteca Virtual em Saúde (BVS), Scientific Electronic Library Online (SCIELO), ScienceDirect.

Assim definidos descritores e palavras-chave, de acordo com Descritores em Ciência (DECS). Na busca das literaturas, usados operadores booleanos "and" respectivamente entre descritores e palavras-chave, empregado filtros entre 2016 a 2021, utilizando descritores em língua portuguesa, inglesa e palavras-chave em português, demostrados em Quadro 1.

Conforme Quadro 2, cruzamento dos descritores e palavras-chave, caracteriza a pesquisa nas bases de dados (PUBMED), (Web of Science), (BVS), (SCIELO), (ScienceDirect) e identifica quais descritores aplicados em cada plataforma.

Serão usados como critérios de exclusão: que não respondiam à pergunta alvo, publicados a mais de 6 anos, fica fora desse critério estudos pioneiros ou de grande importância para comunidade cientifica. Após a investigação, os critérios de inclusão escolhidos, literaturas publicadas nos últimos 6 anos, que tenha acesso em língua portuguesa, inglesa ou espanhol, análise de título, volume, ano, local de publicação, tipo de estudo. Primeira etapa de exclusão segundo os critérios já citados, $\operatorname{logo}$ após $1^{\circ}$ seleção por meio da leitura do título e resumo das literaturas, $2^{\circ}$ exclusão estudos incompletos, artigos similares.

Dessa forma, a quantidade de artigos escolhidos no primeiro momento de pesquisa e montagem da pesquisa para a defesa I, são 151 artigos selecionados das bases de dados eletrônicas, na busca primária para leitura de título e resumo, total de 114 referências excluídas após a leitura de título e resumo, que não fazem parte do critério de inclusão. Referências avaliadas em texto completo 37 no total, eliminadas 15 conteúdos semelhante entre os artigos, total de 22 estudos incluídos para a realização da revisão integrativa de literatura.

Quadro 1- Descritores e palavras-chave.

\begin{tabular}{|l|l|}
\hline Descritores & Palavras-chaves \\
\hline Úlcera por pressão & Lesão por pressão \\
\hline Fatores de risco & Assistência de Enfermagem \\
\hline Cuidados de enfermagem & Prevenção \\
\hline Procedimento terapêutico & Escara de decúbito \\
\hline
\end{tabular}

Fonte: Autores. 
Quadro 2 - Cruzamentos dos Descritores.

\begin{tabular}{|c|c|c|c|c|}
\hline PUBMED & Web of Science & BVS & Scielo & ScienceDirect \\
\hline $\begin{array}{l}\text { "Pressure ulcer" } \\
\text { and "therapeutic } \\
\text { procederus" }\end{array}$ & $\begin{array}{l}\text { "Pressure ulcer" } \\
\text { and "Risk factors" }\end{array}$ & $\begin{array}{l}\text { "Úlcera por } \\
\text { pressão" and } \\
\text { "procedimentos } \\
\text { terapêutico" }\end{array}$ & $\begin{array}{l}\text { "Úlcera por } \\
\text { pressão" and } \\
\text { "procedimentos } \\
\text { terapêutico" }\end{array}$ & $\begin{array}{l}\text { "Pressure ulcer" } \\
\text { and "Risk } \\
\text { factors" }\end{array}$ \\
\hline $\begin{array}{l}\text { "Pressure } \\
\text { ulcer" and "Risk } \\
\text { factors" }\end{array}$ & $\begin{array}{l}\text { "Pressure ulcer" } \\
\text { and "Nursing Care" }\end{array}$ & $\begin{array}{l}\text { "Úlcera por } \\
\text { pressão" and } \\
\text { "Fatores de risco" }\end{array}$ & $\begin{array}{l}\text { "Úlcera por } \\
\text { pressão" and } \\
\text { "Cuidados de } \\
\text { Enfermagem" }\end{array}$ & $\begin{array}{l}\text { "Pressure ulcer" } \\
\text { and "Nursing } \\
\text { Care" }\end{array}$ \\
\hline $\begin{array}{l}\text { "Nursing Care" and } \\
\text { "Pressure ulcer" }\end{array}$ & $\begin{array}{l}\text { "Therapeutic } \\
\text { procedure" and } \\
\text { "Pressure ulcer" }\end{array}$ & $\begin{array}{l}\text { "Cuidados de } \\
\text { Enfermagem" and } \\
\text { "Úlcera por pressão" }\end{array}$ & $\begin{array}{l}\text { "Fatores de risco" } \\
\text { and "Úlcera por } \\
\text { pressão" }\end{array}$ & $\begin{array}{l}\text { "Therapeutic } \\
\text { procedure" and } \\
\text { "Pressure ulcer" }\end{array}$ \\
\hline
\end{tabular}

Fonte: Autores.

\section{Lesão por pressão}

As feridas, são estudadas por equipe multidisciplinar de saúde, com diferentes definições como, escara de decúbito, úlcera por pressão, lesão por pressão (LPP) atualmente mais usual (Lima et al., 2017).

Segundo análise a prevalência de casos de ulceras por pressão são recorrentes, de acordo com estudos da comunidade cientifica, apresentam números de prevalência entre 0,38 a 53,2\%, e incidência 1,9 até 71,6\% em tais localizações, Europa, China, Japão, Estados Unidos, Oriente Médio, Canadá e Austrália. E em relação a realização de tratamento agudo de ulceras por pressão com média de 3,3 até 53,4\% (Loudet et al., 2016).

O National Pressure Ulcer Advisory Panel (NPUAP), em 2007, atualizou a definição de Úlcera por Pressão e classificação, em 2016 a terminologia mudou para Lesão por Pressão (LPP), lesão na pele e/ou tecidos ou em regiões anatômicas de proeminência óssea, fricção, cisalhamento e pressão exercida sobre a pele (Farias et al., 2019).

A gestão hospitalar tem o papel de defender a segurança do paciente, dentre os principais pontos, evitar agravos, e surgimento de processos infecciosos que atingem diretamente a saúde do paciente. Estes eventos adversos, podem acontecer em diferentes setores dos hospitais, tais como, pronto-socorro, unidade de internação, unidade de terapia intensiva (UTI) que recebem o percentual de pacientes mais graves e com lesão por pressão em grau que possam sentir dor, aparecimento da mobilidade prejudicada e acontece em alguns casos o desbridamento dos tecidos da pele, visto como ponto negativo para o enfermeiro e paciente (Oliveira et al., 2016).

A lesão por pressão é causada por uma certa pressão gerada sobre uma área do corpo em junção com outros fatores intrínsecos e/ou extrínsecos, ocasionando lesões na pele, tecidos moles, e tem fator agravantes proeminência óssea devido menor quantidade de tecidos moles ou presença de dispositivos ou monitorização (Jesus et al., 2020).

As lesões por pressão, causam deficiência na integridade da pele o maior órgão do corpo humano, auxilia na manutenção da homeostase corporal, flexível, intacta, uma vez que a mesma sofre lesão podendo ocasionar infecções, na pior das hipóteses causando a amputação de membros e lesão irreversíveis (Murphree, 2017).

As lesões por pressão são classificadas em IV estágios e classificação de lesão por pressão, o estágio I, presença de hiperemia na pele intacta, mais característico em proeminência óssea, por seguinte, o estágio II lesão na derme e epiderme, 
presença de bolhas, abrasão e perda parcial do epitélio e a pele escurecida, úlcera dolorosa causada por exposição das terminações nervosas, em relação ao estágio III caracteriza por perda do tecido dérmico, expondo a gordura subcutânea, sem exposição óssea, tendão e músculos. E o último estágio IV, ocorre perda total de tecido, exposição óssea, músculo e tendão (Farias et al., 2019).

\section{Atuação do enfermeiro frente a prevenção e tratamento de lesão por pressão}

O profissional de enfermagem oferece influência na realização de procedimentos e na ocorrência de lesão por pressão, em pacientes internados. Pesquisas mostram o alongamento das horas de trabalho do profissional de enfermagem em hospitais, e reduzir a equipe de enfermagem não seria viável devido a importância da assistência prestada, em contrapartida pode ocorrer uma queda na qualidade do trabalho desenvolvido, pontos que podem ocasionar maior exposição do paciente e enfermeiro, além de adição de custos extras no tratamento (Oliveira et al., 2016).

A teoria do comportamento, que avalia o comportamento individual e caso não desenvolva um determinado comportamento caracteriza por atitude, essa atitude pode estar relacionada com crenças comportamentais resultado da participação em atividades, e crenças de controle repulsão em envolver-se em certa atividade. O conhecimento desse individuo também é um ponto essencial na avaliação da teoria do comportamento (Heidemann et al., 2007).

A importância da prevenção da lesão por pressão (LP), está correlacionada com a qualidade de serviço, ofertados pelos hospitais e na atenção primária em saúde. Com existência de avanços me saúde, principalmente cientificamente, cuidados com lesão por pressão permanece como um desafio para equipe multidisciplinar e gerencial dos serviços de saúde e familiares (Souza et al., 2020).

Mundialmente, vem sendo estudada a mais de 20 anos, anteriormente nomeada de ulcera por pressão e atualmente descrita como lesão por pressão, as falhas na gestão nacional afetam que poderia acontecer uma melhor gestão em relação a esse agravo. Em diversas situações lesão por pressão é inevitável, mas não justifica os níveis elevados deste agravo em saúde, enfatizando as falhas na produção de estudos científicos a respeito do tema (Souza et al., 2020).

A enfermagem é conhecida por desempenhar ações preventivas das LPPs intra-operatória, comprovadamente em paciente de unidades hospitalares. As lesões por pressão com incidência em pacientes, com mobilidade prejudicada ou imobilidade, má nutrição, fluxo sanguíneo comprometido e neuropatia, observar esses fatores é essencial para a realização de medidas preventivas (Mb et al., 2020).

As medidas de prevenção estão ligadas à utilização de procedimentos para alívio de pressão, e estão ligadas através do conhecimento da equipe de saúde que presta assistência ao paciente, medidas de prevenção é uma preocupação da equipe multiprofissional, e em sua grande maioria pelos profissionais de enfermagem. A equipe de enfermagem, veem realizando melhores atendimentos e comprimento de protocolos buscando a prevenção de lesão por pressão, buscam soluções para realização do cuidado de enfermagem (Freire et al., 2020).

\section{Vulnerabilidade e riscos e lesão por pressão}

A lesões por pressão possui impacto na saúde, incidência mundial, sendo um dos agravos mais comum a pessoa em internação, segundo estudos cerca de 14,3\% e 18,7\% no mundo, e no Brasil incidência em números cerca de 23,1\% e 59,5\%. Estes dados não podem ser generalizados pois se trata apenas de pacientes hospitalizados, em unidade de terapia intensiva UTI, e casa de idosos, as LPPs aumentam o risco de infecções hospitalares, dificultam a recuperação e em consequência eleva o tempo de internação, acarretam mudanças na rotina, além de dor e sofrimento ao paciente e familiar (Santos et al., 2020).

Os riscos relacionados aos pacientes internados e propensos a desenvolver lesão por pressão, são identificados através da utilização da Escala de Braden. Essa escala vem sendo usada desde 1987 no Brasil para avaliação de riscos em paciente, 
podem ser avaliados segundo a escala, estado nutricional, nível de mobilidade, percepção sensorial, fricção, cisalhamento, umidade e grau de atividade física do cliente, verificam-se durante o período de internação, usando esta escala o profissional de saúde diminui o surgimento de LPPs e presta uma assistência de qualidade (Santos et al., 2018).

\section{Resultados e Discussão}

As lesões por pressão é um problema de saúde em todo o mundo, reduz a qualidade de vida do paciente, inferir como o resultado de má qualidade de cuidados de enfermagem, por outro lado, para que aconteça a prevenção e o tratamento e intervenção terapêutica necessário o enfermeiro ter conhecimento, portanto, há uma falha entre a teoria e a prática (Saleh et al., 2019).

A lesão por pressão é causa vulnerabilidade e riscos à saúde dos pacientes, afetam nos indicadores de qualidade da assistência de enfermagem prestadas aos clientes. Dessa forma, evidenciando a importância do profissional de enfermagem realizando educação em saúde, além de prestar assistência nas unidades de saúde, com conhecimento e atualização profissional constante, os cuidados de enfermagem são primordiais e atuam na prevenção de lesão por pressão, um problema de saúde que precisa ser estudado e realizar atualização profissional a respeito desde problema de saúde público.

O estudo realizado com 84 pacientes neurocríticos em Unidade de Terapia Intensiva (UTI), coleta de informações mediante registros de enfermagem, os cuidados mais frequentes observados são de acordo com a análise estatística, a aplicação da Escala de Coma de Glasgow 87,8\%, Escala de Agitação e Sedação Richmond 84,3\%, avaliação das pupilas 79,7\%, cabeceira elevada a $30^{\circ} 100 \%$, monitorização dos sinais vitais 46,4\% e avaliação da Escala de Braden 36,9\%, tais evidenciam os cuidados de enfermagem prestados em UTI (Caciano et al., 2020).

Estudos apontam que cerca de $41,7 \%$ dos enfermeiros possuem falha no conhecimento sobre prevenção de lesão por pressão, e precisam se aprimorar em relação ao tema. Cerca de $20 \%$ dos enfermeiros responderam corretamente os cuidados em relação a reposicionamento de pacientes imóveis, estas lacunas são vivenciadas mundialmente, além da não frequente atualização dos enfermeiros (Bns, 2020).

A lesão por pressão tem várias causas, dentre elas podemos citar causas intrínsecos e/ou extrínsecos, causando o adoecimento no paciente, consequentemente ocasionando alterações na pele e por seguinte alterando a qualidade de vida, e essas lesão por pressão servindo como porta de entrada para possíveis infecções.

\section{Conclusão}

Conclui-se a necessidade de maiores estudos a respeito de Lesão por Pressão que veem evidenciando com embasamento cientifico a incidência de LPPs mundialmente, dados em números. A vulnerabilidade e riscos à saúde dos pacientes, afetam nos indicadores de qualidade da assistência de enfermagem prestadas aos clientes, portanto, a importância da equipe multidisciplinar em destaque a Enfermagem ter conhecimento em relação a LPPs, e cuidados é o primeiro passo para realização de medidas de prevenção e tratamento, evitando agravos a saúde do paciente.

São expostas as conclusões deste artigo, e as recomendações para a continuidade dos trabalhos nesta área de estudo. O presente trabalho teve o objetivo de informar a população científica, a respeito da incidência de LPP’s e o impacto na saúde do paciente e impacto na rotina de trabalho do profissional enfermeiro, e por consequência enfatizando a necessidade de atualização profissional sobre o tema LPP’s, além da importância de novos estudos a respeito. Por fim, sugere-se também a realização da atualização profissional dos enfermeiros, como por exemplo, especialização, esta sugestão poderia ser aplicada através das unidades de ensino superior. 


\section{Agradecimentos}

À Deus, por ter me capacitado, proporcionando sabedoria e força em todos os dias durante este percurso. Aos meus pais, João Mendes e Lucilene Neres, ao apoio incondicional, amor de pais, respeito, incentivo, cuidado, proteção, por me proporcionar oportunidade de estudar, os senhores são a razão de todas as minhas realizações. Agradeço, à minhas irmãs $\operatorname{Dr}^{\mathrm{a}}$ Karina Neres e Dr ${ }^{a}$ Kely Neres, pelo companheirismo e amor que me fez seguir em frente.

Aos meus colegas e professores da Faculdade dos Carajás em Marabá-Pará, professora enf. me. Percília Augusta Santana, as minhas amigas e colegas de graduação em enfermagem, enf. Eugene Adelaide, enf. Marlete Ibiapina, enf. Poliana Carvalho, por partilhar conhecimentos teóricos, práticos e parceria nesses 4 anos de vivência, nos anos de 2015 a 2019.2.

A Faculdade Integrada Carajás FIC em Redenção-Pará, no período de tempo de 2021.1 a 2021.2, a todos, pelo acolhimento e responsabilidade com a finalização da minha formação profissional, em especial aos professores enf. Fabiana Loureiro, Dr. Jânio Sousa Santos, Dr ${ }^{\mathrm{a}}$ Danielle Das Neves Bespalhok e orientador do trabalho de conclusão de curso enf. Me. Marcos Vinícius Ferreira dos Santos. Agradeço aos meus professores e colegas do curso de medicina da Faculdade de Ensino Superior da Amazônia Reunida- FESAR em Redenção-Pará, por incentivar a não desistir das minhas metas durante essa jornada difícil de conciliar duas graduações.

À todos os profissionais e pacientes do Hospital Central de Marabá, Hospital Regional Público do Araguaia, Hospital Materno Infantil Dr. Pedro Paulo Bacauí, em especial a enf. Magda Bispo, somar em conhecimentos teóricos e práticos, e compartilhar sua experiência profissional. Agradeço a todos aqueles que de alguma forma contribuíram para a realização deste trabalho.

\section{Referências}

BNS, L. J. (2020). Nurses' knowledge, atitudes, and behaviours related to pressure injury prevention: A large-scale cross-sectional survey in mainland China. Journal of Clinical Nursing. 29 (1), 3311-3324. https://onlinelibrary.wiley.com/doi/full/10.1111/jocn.15358.

Brasil. (2013). Ministério da Saúde. Portaria No 529, de $1^{\circ}$ de Abril de 2013, Institui O Programa Nacional de Segurança do Paciente (PNSP). Diário Oficial da União, Brasília. Disponível em: Ministério da Saúde (saude.gov.br).

Caciano, K. R. P. S., Saavedra, J. L. I., Monteir, E. L., Volpáti, N. V., Amaral, T. L. M., Sacramento, D. S. \& Prado, P. R. (2020). Intervenções de Enfermagem para pacientes neurocríticos. Revista de Enfermagem UFPE on-line. 14 (e243847), 1-9.

Chavaglia, S. R. R., Ohl, R. I. B., Gamba, M. A., Petri, V. \& Costa, M. T. F. (2016). Feridas prevenção, causas e tratamento. 1. ed. Rio de Janeiro: Santos Ed. 40-52.

Estrela, C. (2018). Metodologia científica: ciência, ensino, pesquisa. 3.ed. Porto Alegre: Artes Médicas. 201-205.

Farias, A. D. A., Leal, N. T. B., Travassos, N. P. R., Farias, A. J. A., Nobre, A. M. D. \& Almeida, T. C. F. (2019). Ocorrência de lesões por pressão em unidade de terapia intensiva de um hospital universitário. Revista Nursing. 22 (253), 2927-2931.

Freire, D. A., Oliveira, T. S., Souza, N. R., Santos, F. M. E., Santos, K. S. \& França, M. J. D. M. (2020). Variáveis associadas à prevenção das lesões por pressão: conhecimento para o cuidado de enfermagem. Revista Online de Pesquisa Cuidado é Fundamental. 12 (1), $1172-1178$.

Gamston, J. (2019). Pressure induced skin and tissue injury in the emergency department. Emergency Medicine Journal. 36 (1), $631-634$.

Heidemann, L. A., Araujo, I. S. \& Veit, E. A. (2012). Um referencial teórico-metodológico para o desenvolvimento de pesquisas sobre atitude: a Teoria do Comportamento Planejado de Icek Ajzen. Revista electrónica de investigación en educación en ciências REIEC. 7 (1), 1-10.

Jesus, M. A. P., Pires, P. S., Biondo, C. S. \& Matos, R. M. (2020). Incidência de lesão por pressão em pacientes internados e fatores de risco associados. Revista Baiana de Enfermagem. 34 (e), e36587.

Lima, R. V. K. S., Coltro, P. S. \& Júnior, J. A. F. (2017). Terapia de pressão negativa para o tratamento de feridas complexas. Revista do Colégio Brasileiro de Cirurgiões. 44 (1), 81-93.

Loudet, C. I., Marchena, M. R. M., Fernández, S. L., Romero, M. V., Velenzuela, G. E., Herrera, I. E., Ramírez, M. T., Palomino, S.R., Teberobsky, M. V., Tumino, L. I., González, A. L., Reina, R. \& Estenssoro, E. (2016). Diminuição das úlceras por pressão em pacientes com ventilação mecânica aguda prolongada: um estudo quase-experimental. Revista Brasileira de Terapia Intensiva. 29 (1), 39-46.

Mb, F. J., Mm, Y. F., Mb, Y. Z., Mb, J. Y., Mm, K. Z. \& Mm, H. L. (2020). Effect of intraoperative pressure ulcer preventive nursing on inflammatory markers in patients with high-risk pressure ulcers. Medicine Journal. 99 (20) 1-4.

Murphree, R. W. (2017). Impairments in Skin Integrity. Nursing Clinics of North America. 52 (3), 405-417. https://doi.org/10.1016/j.cnur.2017.04.008/. 
Oliveira, A. C., Garcia, P. C. \& Nogueira, L. S. (2016). Carga de trabalho de enfermagem e ocorrência de eventos adversos na terapia intensiva: revisão sistemática. Revista Escola de Enfermagem, USP. 50 (4), 683-694.

Russell, C. L (2005). An overview of the integrative research review. Progress in Transplantation. 15 (1), 8-13, 2005. https://www.researchgate.net/publication/7898657_An_overview_of_the_integrative_research_review.

Saleh, M. Y. N., Papamnikolaou, P., Nassar, O. S., Shahin, A. \& Anthony, D. (2019). Nurses' knowledge and practice of pressure ulcer prevention and treatment: Na observation study. Journal of Tissue Viability. 1 (1), 1-8. https://doi.org/10.1016/j.jtv.2019.10.005.

Santos, C. C.; Hamburgo, V. P., Santana, T. S., Sousa, A. R. \& Carvalho, E. S. S. (2020). Educação em serviço para a prevenção de lesão por pressão através do planejamento estratégico situacional. Revista de Divulgação Científica Sena Aires REVISA. 9 (4), 773-83.

Santos, L. R. C. \& Lino, A. I. A. (2018). Riscos de lesão por pressão: aplicação da Escala de Braden em terapia intensiva. Revista Estima. 16 (e0818), 1-7. https://www.revistaestima.com.br/estima/article/download/443/pdf_1/1208.

Souza, M. T., Silva, M. D. \& Carvalho, R. (2010). Revisão integrativa: o que é e como fazer. Einstein (São Paulo), 8 (1), 102-106. https://journal.einstein.br/pt-br/article/revisao-integrativa-o-que-e-e-como-fazer/.

Souza, M. C., Loureiro, M. D. R. \& Batiston, A. P. (2020). Cultura organizacional: prevenção, tratamento e gerenciamento de risco da lesão por pressão. Revista Brasileira de Enfermagem. 73(3), e20180510. https://www.scielo.br/j/reben/a/nTWn65rm7y3 YyFwV9dXpd8x/?lang=pt\&format=pdf.

Woodhouse, M., Worsley, P. R., Voegeli, D., Schoonhoven, L. \& Bader, D. L. (2019). How consistent and effective are current repositioning strategies for pressure ulcer prevention?. Applied Nursing Research. 48, 58-62. 